\title{
THE MARITAL STATUS AS PREDICTOR OF DIFFERENCES IN TIME PERSPECTIVE AND OPTIMISTIC-PESSIMISTIC ATTITUDES AMONG WOMEN
}

\author{
Natasha Angelova \\ South-West University "Neofit Rilski", Bulgaria \\ E-mail: natasha_v@swu.bg \\ Magdalena Trencheva \\ Blagoevgrad, Bulgaria \\ E-mail: magi79@abv.bg
}

\begin{abstract}
The search for relations, dependencies, and influences between marital status and mental phenomena outlines an accurate and clear picture not only of the family status as a social phenomenon but also as a mental field, where our awareness as individuals is directly or indirectly linked. In this sense, the differences among women with different marital context and the optimistic and pessimistic attitudes as well as the expectations regarding the past, present, and future that were investigated in this research.

Four different marital status groups were identified and are shown the differences in time perspective and optimistic-pessimistic attitudes between them. The survey was conducted with 140 women aged between 32 and 67 years $M=41.09 S D=6.58$. The research methods are Method of evaluation of optimism and negative expectations, published by Velichkov and Radoslavova (2005) and Time perspective Questionnaire by Philip Zimbardo, adapted by Slavchov and Virmozelova (2008).

The results verify the supposition that there are statistically significant $(p<.05)$ differences between the four groups of women with different marital status, with regard to their attitudes to optimism and pessimism as well as to their time perspective. The group of married women shows the highest result in Past-positive time perspective and Future time perspective, in comparison with the divorced that have the lowest scores on the same factors Past-positive time perspective and Future time perspective. At the same time, the divorced women experience the highest level of optimism compared to the other groups.
\end{abstract}

Keywords: time perspective, optimism - pessimism, marital status.

\section{Introduction}

The aging period considered in this study is significant, as it is the most dynamic in the change of the marital status. According to Erik Erikson (1982), this period of human life is extremely important for enriching the cultural environment, caring for others and paying attention to the needy relatives. The inability for active family involvement leads to a crisis that the author believes to lead to alienation and inability to discover a meaning both in activities and in communicating and caring for others. At this stage, people are both trainers and trainees- while „rowing with full 
handfuls from the fountain of life" and learning their life lessons, simultaneously actively helping others to make their first steps in life and "to learn how to learn from life". At this stage, creativity Vol. 11, No. 1, 2017 is important as long as it provides and maintains the ideals, principles and supports mental health. If the field of productivity, commitment does not expand people become a victim of boredom and stagnation.

\section{Marital Status and Family}

Marital status is a phenomenon which nowadays does not enjoy any special interest neither in popular nor scientific psychological literature. To a certain extent, this is probably due to the scientists' striving to support "old" perceptions, to look for the reasons in family relationships without looking for new perspectives that reflect the contemporary rhythm in partner relationships. The contemporary view on the marital status of individuals should reflect on one hand the criteria for the dynamics of development of society, and on the other - on purely personal level, to reflect the capabilities of the individual to bear in himself the conflict between the imposed and familiar patterns of family life in the society and personal comfort in pursuing subjective values and satisfying his own interests.

The family is an indispensable environment for human existence. It is the bio-social structure in which man develops. Through the family, the continuity in the social development is ensured. The marriage is thought as a source of well-being that brings social and personal benefits in human life (Wade etc. 2013). Modern society is witnessing a gradual change in family perceptions. The marital relationship performs not only biological but also the social reproduction of people and it depends on the economic, technological, political and other principles of functioning of the society. Hooghe and Vanhoutte (2011) reported the stronger social ties, even after adjusting for levels of optimism people experienced, the greater the levels of life satisfaction are. According to Wilson and Oswald (2005), the married persons seem to be happier than those who cohabit, who are themselves happier than single individuals. The married individual also has better physical and psychological health, longevity than the never married, that in turn does better than the divorced, separated and widowed. In terms of human psychosocial evolution human beings are learning beings - therefore, each generation transmits its experience to the next, assisting it in its identity and building up skills and habits to deal with the life events. The uniqueness of the mankind consists of the fact that the engagement with the developing generation and his upbringing is extended at the time. This gives meaning to the concrete human existence. Marriage may itself lead to a surge in the expectations of each individual about their lives. This has been called the protective effect of marriage. Some papers (Kim \& Mckenry, 2002) reveal less depression among married people than among cohabiters. In another research study (Kohler et al., 2004) the authors concluded the cohabiters are shown to be the group with the highest alcohol abuse and the results shown significant positive effects upon well-being for individuals in current cohabitation or marriage relationships relative to being single.

\section{Optimism and Pessimism}

Optimistic individuals are more likely to be positive about events in daily life and tend to have more frequently protective attitudes. They have an adaptive management of personal goals and development to compare with pessimists.

In this order, problem-solving is associated with psychological dimensions of the phenomenon of "optimism". Pessimism and optimism are considered primarily as cognitive constructs associated with the activity or passivity toward the future, underestimating the volitional factor in both attitudes. Minaker (2001) claims that pessimism and optimism are primary temperamental categories and, in this sense, have a considerable predestination. Although he makes the notion that the more socially tied you are, the more educated you are, the better you eat and sleep, the easier it will be to keep an optimistic view of things, despite your temperamental predispositions (Minaker, 2001).

Being optimistic in the full sense of the term means to show the following tendencies: 
- to have a positive attitude towards the future;

- to set very specific task goals;

- to show perseverance/persistence to the difficulties;

- to believe in personal effectiveness /self - efficacy;

- to give yourself predominantly positive self-assessments /self-evaluation;

- to believe that you can control the situations of your life;

- to have a positive explanatory style for the events in your life;

- to learn from your mistakes;

- to believe in the goodwill and goodness of others;

- to have flexible coping strategies.

But to be pessimistic means to do the opposite of the above dispositions, but also with some specifics:

- to give up on the difficulties;

- to attribute your failures to the lack of ability;

- to focus on your emotions when it's difficult for you;

- to have a philosophy of life, justifying inaction, negativity, and cynicism because of the bad organization of the world.

The roots of optimism are in the peculiarities of individual development in childhood and the practices of parental care and family education. Negative expectations are secondary, they are formed in adulthood through the gradual and inevitable accumulation of negative life experiences.

\section{Time Perspective}

The time spent with family, relatives, and friends enhances people's optimistic attitudes in their lives and future perspectives. There are some researchers who studied the link between time orientation and optimism as a predictor of well-being, health behavior, and problem-solving strategies. A person's orientation to the time, which is the preferred direction of the individual's thoughts and actions to the past, present or future (DeVolder, 1979), has a dynamic impact on his judgments, decisions, and actions. At the level of the individual psyche, time is included exactly through the awareness of the realized possibility and necessity of doing certain actions towards the coming changes. Cartensen and colleagues (Carstensen, Isaacowitz \& Charles, 1999) suggest that human perception of time plays an important role in choosing and pursuing social goals with important emotional, cognitive and motivational effects.

The Time perspective is an individual-difference variable that is studied through its relation with a variety of psychological concepts. The time orientation or originally named by Zimbardo "perspective" is perceived as "an unconscious process in which the continuous flow of personal and social experience is assigned to temporary categories or time frames that help to create order, coherence, and meaning for these events" (Zimbardo \& Boyd, 1999). Philip Zimbardo and John Boyd dedicate more than 30 years of research, related to the time perspective as a construct. The psychological time is expressed in what is the perception and the attitude of people towards their past, present and future experience. Knowledge, competencies, creativity, and skills are needed to create a test tool or experiment that can reveal accurately, clearly and correctly the psychological notions of time.

The author of "Time Perspective Inventory", Philip Zimbardo, identifies a total of 5 categories of time perspective: past-negative, past-positive, present-fatalistic, present-hedonistic, future.

Past-negative: This attitude reflects a generally negative and pessimistic view of the past. This negative attitude to the past can be attributed to actual events that have been experienced as traumatic or unpleasant or may be due to a negative reconstruction of past events. Often this attitude might be a mixture of both. A person with a predominantly negative attitude to the past may often turn out to be living through the unpleasant past experience over and over again, thus experiencing the disappointment or trauma again. 
Present - hedonistic: This attitude characterizes people „living for the moment“. Thus, riskVol. 11, No. 1, 2017

taking and hedonistic behavior are connected with people associated with this way of thinking. This attitude suggests orientation to the present pleasure to such extent that very little concern is expressed about future consequences.

Future: People associated with this attitude are focused on future goals and awards. This attitude is associated with a focus on the future where the current situation is interpreted in terms of future consequences. A large number of previous studies have shown that high levels of future time perspective are related to marriage, to higher incomes and to achieve a high level of education (Gonzalez \& Zimbardo, 1985). The Perspective of the Future is the way a person integrates the future into his mental present. It depends and is built mostly on the way we set our goals. The two main features of the future perspective have a major role in building our motivation and include the ways in which, a person, using his past experience specifies his vague desires in certain goals, plans, and concrete projects for implementation. It turns out that the orientation towards the future predicts healthier behavior. Smokers and substance abusers are less future-oriented. Also, people with a predominant orientation towards the future are more mature in terms of their career development, and risky sexual behavior is less common.

Past - Positive: People with a positive attitude have a tendency to reflect on past experiences. However, unlike the past-negative attitude, this tendency generates feelings of warmth and sentimentality.

Present - fatalistic: This attitude reflects the predominantly helpless and hopeless attitude towards life and the future in general. People who think this way often feel out of control in the situations they are in.

According to Zimbardo (Zimbardo \& Boyd, 1999), each individual time perspective is not directly related to others. Each person has a unique combination of time perspectives. The more meaningful is the life of a person, the more positively he perceives his present. With a high degree of meaningfulness, the orientation of the positive past prevails (Zimbardo, 2015) and, in case of a lower degree - the negative past is predominant.

\section{Aims and Hypothesis}

The present research aims to determine whether the marital status has an influence on the dual cognitive attitudes (optimism-pessimism) and on individual's time perspectives. According to this were also explored the differences between four group marital status in terms of optimismpessimism as well as the time orientation to the past negative or positive, present fatalistic or hedonistic and future.

The main hypothesis is that the marital status influences the women`s optimistic-pessimistic attitudes and their time perspectives. There are statistically significant differences between the four groups of marital status in terms of women 's attitudes to optimism and pessimism as well as their time perspectives.

\section{Methodology of Research}

The analysis was conducted using SPSS 16 . The data collected were analyzed by One Way ANOVA to determine whether there are any statistically significant differences between the means of independent groups (the marital status is divided into four groups). An Eta squared, $\eta^{2 \prime}$ has been used to effect size. It ranges between 0 and 1 (.02 small; $.13 \sim$ medium; $.26 \sim$ large).

\section{Research Instruments}

Method of evaluation of optimism and negative expectations. The questionnaire was published by Velichkov and Radoslavova (2005). It consists of 17 items divided into two subscales: Optimism where 10 items are included, Negative expectations containing the remaining 7 items. The evaluation of the questions is performed on a 5-point Likert scale: 1 - Completely Disagree; 2 - Disagree, 3 - Partly agree, partly disagree, 4 - Agree, 5 - Totally agree. 
Natasha ANGELOVA, Magdalena TRENCHEVA. The marital status as predictor of differences in time perspective and optimisticpessimistic attitudes among women

Kaiser-Meyer-Olkin Measure and Bartlett's test, which evaluates whether the distribution of values is adequate for conducting a factor analysis gives a good result $\mathrm{KMO}=.754 \mathrm{p}<.001$ (Dzhonev, 2004, pp.77).

Time Perspective Questionnaire by Philip Zimbardo, adapted by Slavchov and Virmozelova (2008). This questionnaire consists of 56 items distributed in 5 subscales: Past-negative, which includes the negative attitude towards the past and the negative memories of it. This subscale includes 10 items. Past positive subscale, this includes 9 items. Present fatalistic subscale in which 9 items are included. Present hedonistic time perspective subscale where 15 items are present. Future time perspective, this subscale includes 13 items. Answering the questions is done through a 5-point Likert scale: 1 - Does not apply to me at all; 2 - Does not apply to me to a large extent 3 - I cannot decide, 4 - To a great extent it applies to me and 5 - It is completely relevant to me.

Kaiser-Meyer-Olkin Measure and Bartlett's test is adequate for conducting a factor analysis, which gives a sufficient result $\mathrm{KMO}=.557 \mathrm{p}<.001$ (Dzhonev, 2004, pp.77).

\section{Participants}

The survey was conducted between March and May 2017. Both questionnaires were given to the respondents through a non-government organization for family and child care and completed simultaneously following the instructions, and the confidentiality of the surveyed persons was observed when registering the forms.

Table 1. Marital status, number, and percent of the participants.

\begin{tabular}{ccc}
\hline Marital status & $\mathbf{N}(\mathbf{1 4 0 )}$ & $\%$ \\
\hline Singles & 41 & 29.30 \\
Cohabiters & 8 & 5.70 \\
Married & 83 & 59.30 \\
Divorced & 8 & 5.70 \\
Widowed & 0 & 0 \\
\hline
\end{tabular}

According to the aim of research participants that have been studied are only women $(\mathrm{N}=140)$ aged between 32 and 67 years $(M=41.09 \mathrm{SD}=6.58)$. The Participants were divided into four groups because there weren' $t$ any widowers in the sample.

\section{Results of Research}

The presented statistical analysis in Table 2 shows a level of significance, per three out of six factors, that allows interpreting the effect size and intergroup differences in the way they are given in the hypothesis. These differences stand out more clearly from Table 2 . The values obtained with a coefficient of significance $\mathrm{p}<.05$ showed that there are statistically significant differences between the four groups of women with different marital status, with regard to their attitudes to optimism and pessimism as well as to their time perspective.

Table 2. Means, standard deviation, f-criterion, eta-squared $\left(n^{2}\right) n=140$.

\begin{tabular}{cccccccccc}
\hline Factor & Marital status & $\mathbf{N}$ & Mean & $\begin{array}{c}\text { Standard } \\
\text { deviation }\end{array}$ & Minimum & Maximum & $\mathbf{F}_{\mid \text {dafl }}$ & $\mathbf{P}$ & $\boldsymbol{\eta}^{2}$ \\
\hline \multirow{4}{*}{ Optimism } & Singles & 41 & 35.97 & 7,11 & 21.00 & 50.00 & & & \\
& Cohabiters & 8 & 32.12 & 4,94 & 27.00 & 42.00 & $2.830_{|3|}$ & .04 & .059 \\
& Married & 83 & 37.97 & 5,74 & 17.00 & 50.00 & & & \\
& Divorced & 8 & 38.00 & 5,58 & 27.00 & 43.00 & & & \\
\hline
\end{tabular}




\begin{tabular}{|c|c|c|c|c|c|c|c|c|c|}
\hline Factor & Marital status & N & Mean & $\begin{array}{l}\text { Standard } \\
\text { deviation }\end{array}$ & Minimum & Maximum & $F_{|d f|}$ & $P$ & $\eta^{2}$ \\
\hline \multirow{4}{*}{$\begin{array}{l}\text { Negative } \\
\text { expectations }\end{array}$} & Singles & 41 & 19.73 & 5,34 & 9.00 & 28.00 & \multirow{4}{*}{$2.348_{|\beta|}$} & \multirow{4}{*}{.07} & \multirow{4}{*}{.049} \\
\hline & Cohabiters & 8 & 23.50 & 3,89 & 18.00 & 29.00 & & & \\
\hline & Married & 83 & 19.06 & 4,30 & 11.00 & 29.00 & & & \\
\hline & Divorced & 8 & 18.75 & 4.49 & 15.00 & 28.00 & & & \\
\hline \multirow{4}{*}{$\begin{array}{c}\text { Past } \\
\text { negative }\end{array}$} & Singles & 41 & 26.19 & 7.35 & 9.00 & 42.00 & \multirow{4}{*}{$1.528_{|\beta|}$} & \multirow{4}{*}{.21} & \multirow{4}{*}{.033} \\
\hline & Cohabiters & 8 & 25.50 & 5.18 & 20.00 & 36.00 & & & \\
\hline & Married & 83 & 27.60 & 6.96 & 13.00 & 41.00 & & & \\
\hline & Divorced & 8 & 22.62 & 5.78 & 12.00 & 30.00 & & & \\
\hline \multirow{4}{*}{$\begin{array}{l}\text { Past } \\
\text { positive }\end{array}$} & Singles & 41 & 29.14 & 5.04 & 19.00 & 39.00 & \multirow{4}{*}{$3.424_{|3|}$} & \multirow{4}{*}{.01} & \multirow{4}{*}{.070} \\
\hline & Cohabiters & 8 & 28.37 & 2.92 & 23.00 & 33.00 & & & \\
\hline & Married & 83 & 29.85 & 5.69 & 16.00 & 41.00 & & & \\
\hline & Divorced & 8 & 23.50 & 5.95 & 17.00 & 36.00 & & & \\
\hline \multirow{4}{*}{$\begin{array}{c}\text { Present } \\
\text { hedonistic }\end{array}$} & Singles & 41 & 48.17 & 8.61 & 31.00 & 64.00 & \multirow{4}{*}{$.395_{|3|}$} & \multirow{4}{*}{.75} & \multirow{4}{*}{.009} \\
\hline & Cohabiters & 8 & 47.37 & 11.74 & 24.00 & 60.00 & & & \\
\hline & Married & 83 & 46.27 & 9.31 & 23.00 & 70.00 & & & \\
\hline & Divorced & 8 & 48.00 & 14.29 & 31.00 & 67.00 & & & \\
\hline \multirow{4}{*}{$\begin{array}{l}\text { Present } \\
\text { fatalistic }\end{array}$} & Singles & 41 & 27.41 & 6.65 & 13.00 & 40.00 & \multirow{4}{*}{$1.295_{|3|}$} & \multirow{4}{*}{.27} & \multirow{4}{*}{.028} \\
\hline & Cohabiters & 8 & 26.00 & 6.45 & 19.00 & 37.00 & & & \\
\hline & Married & 83 & 29.26 & 5.87 & 13.00 & 44.00 & & & \\
\hline & Divorced & 8 & 28.25 & 6.08 & 21.00 & 38.00 & & & \\
\hline \multirow{4}{*}{ Future } & Singles & 41 & 40.04 & 6.74 & 28.00 & 54.00 & \multirow{4}{*}{$5.733_{|\beta|}$} & \multirow{4}{*}{.01} & \multirow{4}{*}{.110} \\
\hline & Cohabiters & 8 & 42.25 & 5.87 & 33.00 & 53.00 & & & \\
\hline & Married & 83 & 43.50 & 6.32 & 28.00 & 57.00 & & & \\
\hline & Divorced & 8 & 34.75 & 10.05 & 19.00 & 53.00 & & & \\
\hline
\end{tabular}

The empirical data proves that the marital status has an impact on the following three factors: Optimistic attitudes $\mathrm{F}_{|3|}=2.830 \mathrm{p}<.05$ with medium effect size $\eta 2=.059$; Past-positive perspective $\mathrm{F}_{|3|}=3,424 \mathrm{p}<.05$ with medium effect size $\eta 2=.070$ and Future perspective $\mathrm{F}_{|3|}=5.733 \mathrm{p}<.05$ with medium effect size $\eta 2=.110$.

According to the statistical analysis used for detecting differences, the hypothesis is partially proved. The reported results of Eta-squared describe the marital status as an influential factor in the women's optimistic-pessimistic attitudes and their time perspectives.

Optimism with a value of $\mathrm{F}_{|3|}=2.830 \mathrm{p}<.05$ shows that women with different marital status have a different degree of optimism. It is noticeable that the divorced women have the highest mean score $\mathrm{M}=38.00 \mathrm{SD}=5.58$ on this factor. In contrast, cohabiters have the lowest mean value in terms of optimism $\mathrm{M}=32.12 \mathrm{SD}=4.94$. Here should be kept in mind, that in these two groups there are only 8 women and the comparison of their mean value with the other groups: Singles $(N=41) M=$ 35.97 $\mathrm{SD}=7.11$ and Married $(\mathrm{N}=81) \mathrm{M}=37.97 \mathrm{SD}=5.74$, should not be perceived categorically. But a comparison with the mean value of the divorced, who are also 8 respondents just as the cohabiters, shows us a very interesting result. Although the divorce is a kind of final stage in the evolution of a relationship, the divorced women experience high levels of optimism.

The group of married women shows the highest result $\mathrm{M}=29.85 \mathrm{SD}=5.69$ on the Past-positive time perspective scale $\mathrm{F}_{|3|}=3.424 \mathrm{p}<.05$. Within the expected, the divorced women group has the lowest score on this factor. This result in the context of the previous one for the high mean of the divorced on optimism scale, it seems it could be said with some certainty, that the lack of fixation in the past of the divorced women helps them to develop a strategy, which is not directed towards 
Natasha ANGELOVA, Magdalena TRENCHEVA. The marital status as predictor of differences in time perspective and optimisticpessimistic attitudes among women

mental repetition of past experiences, and their confrontation with dissatisfaction in a past period of time has brought a sense of coping and confidence in one's own abilities to overcome difficult situations. The highest mean value $\mathrm{M}=29.85 \mathrm{SD}=5.69$ on this factor have married women. The married women that were studied are probably still at the stage of determining the experiences and shaping of the marital roles, so the accumulated pleasant emotions are still at the top of the memories list.

On the third factor with significant differences, „Future time perspective” $\mathrm{F}_{|3|}=5.733 \mathrm{p}<.05$, again the married women, compared to other groups, $\mathrm{M}=43.50 \mathrm{SD}=6.32$ most often are headed toward the future. A strong impression, however, makes the fact that the mean value of the divorced women is significantly lower $\mathrm{M}=34.75 \mathrm{SD}=10.05$ compared to all other groups in terms of future orientation.

No significant differences between the other factors (Negative expectations, Past- negative time perspective, Present-hedonistic, and Present-fatalistic time perspective) on the grouping variable marital status were found.

\section{Discussion}

We postulated the marital status influences the women 's optimistic-pessimistic attitudes and their time perspectives. And the results obtained partially proved the hypothesis. Karney (2010) points out those individuals who estimate themselves as happy overall, have a greater ability to highlight the positive attributes of their marriage or relationship. Schneider and colleagues (2012) state that married people who exchange optimism will experience greater success in problem-solving than do pessimists. The authors of the methodology Optimism and Negative Expectations, Radoslavova and Velichkov (2005) report, that optimism and pessimism are not opposite to one another but relatively independent, they are not allocated as opposing trends on one axis. The findings represented here on the Optimism scale argue their admission and this is surprising to the extent that the initial conviction was that the highest values of optimism would have women in cohabitation and marriage. Considering the assumption that in an active relationship with a partner, ideas and expectations for life are more positive and optimistic. One explanation is probably that after a final end in an intimate relation, people tend to focus on life with an optimistic attitude, that every unpleasant thing they fight off is bearing power and a sense of coping with the new challenges. While cohabiters have two different perspectives, on one side, this position brings the feeling of freedom and comfort because of the lack of annoying conventions in property matter, but on the other hand the lack of a clear boundary imposed by society does not confront personal needs and frustrations, which leaves the person in a moratorium on the development of partner interrelationships.

The research attention was also driven by the problem of defining the framework of mental time. Mental time is formed on the basis of the identification of causal links between major events in people's lives. This determinacy of causality has its specificity - it is available and defined both between the events of the present by the events of the past, thus determines the present from the future, i.e. from the goals and predicted results of the life activity, similar relationships reported in his studies also Zimbardo (2008).

The findings here about the married women, who have a look toward the positive past and the future, prove the Zimbardo's (1999) hypothesis that people with a positive experience in the past tend to see positive prospects for their future, so it has been confirmed also in the present study. In contrast, the divorced women direct their thoughts towards the future considerably less than the women of the other groups. According to Sobol-Kwapinska and Jankowski (2015) when the people experience high intensity of Past-Positive and Future and low intensity of Past-Negative and Present-Fatalistic it helps to adopt a flexible strategy to different conditions of life and effective performance of tasks.

The relationship between the three-time patterns (past, present, and future) is determined by the psychological past (which is a set of realized connections), psychological present these links unite the events of the chronological past and the events of the future, so the psychological future represents the potential relations, the realization of which has not yet begun because they bring together the supposed events of the chronological future. 
One important novelty of this study is the accent of the marital status as a predictor of the perceives and reacts to the world. The family context in which the people are, in turn, determines expectations (positive or negative), as well as the readiness and skill to interact with the environment, to get to know their selves and to develop their abilities.

The knowledge of the time perspective attitudes, the optimistic and pessimistic expectations, and the marital status gives the people opportunity to develop their selves within the boundaries defined by the societies and our own capabilities to realize our potentials.

With regard to the limitations of this study, it is important to stress that we include only one demographic indicator, among the many possible individual dimensions that could have been sampled. Further researches on this topic might benefit by expanding the pool of demographic markers in an effort to optimize the prediction of future orientation because of the time perspective dimensions, as well as the dual cognitive attitudes (optimism and pessimism), depending on the group which is investigated (Ryack, 2012).

\section{Conclusions}

In this research the importance of marital context was justified. The outcomes suggest the marital status as a predictive dimension of the optimism and individuals time perspectives. A practical usage of these findings could be to help people improve their everyday life functioning in their contact with partners and mates, however, to make positive evaluations regarding their future and strategies to tackle difficulties. It is important to notice that the participants that have been investigated are adults and not students. It makes sense to use the results as the direction in counseling and social work with families.

\section{References}

Carstensen, L. L., Isaacowitz, D. M., \& Charles, S. T. (1999). Taking time seriously: A theory of socioemotional selectivity. American Psychologist, 54, 165-181.

Dzhonev, S. (2004) Sotsialna psihologiya [Social psychology], Vol. 5. Sofia.

DeVolder, M. M. de (1979). Time orientation: A review. Psychologica Belgica, 19, 61-79.

Erikson, E. (1982). The life cycle completed. New York: Norton.

Gonzales, A., \& Zimbardo, P. G. (1985). Time in perspective: A Psychology Today survey report. Psychology Today, 19, 21-26.

Hooghe, M., Vanhoutte, B. (2011). Subjective well-being and social capital in Belgian communities: The impact of community characteristics on subjective well-being indicators in Belgium. Social Indicators Research, 100 (1), 17-36.

Karney, B. (2010). Keeping marriages healthy, and why it's so difficult. Retrieved from http://www.apa.org/ science/about/psa/2010/02/sci-brief.aspx.

Kim, H. K., Mckenry, P. C. (2002). The relationship between marriage and psychological well-being. A longitudinal analysis. Journal of Family Issues, 23 (8), 885-911.

Kohler, H. P., Behrman, J. R., \& Skytthe, A. (2004). "Partner + Children = Happiness? An assessment of the effect of fertility and partnerships on subjective well being". The University of Pennsylvania.

Minaker, K. L. (2001). An interview “Anti-Aging or Successful Aging”. Tufts University Health \& Nutrition Letter, 19 (2), 1-2.

Pierce, C. A., Block, C. A., \& Aguinis, H. (2004). Cautionary note on reporting eta-squared values from multifactor ANOVA designs. Educational and Psychological Measurement, 64 (6), 916-924.

Radoslavova, M., \& Velichkov, A. (2005). Metodi za psihodiagnostika [Methods of psychodiagnostics]. Sofia: Pandora Prim.

Ryack, K. (2012). Evidence that time perspective factors depend on the group: Factor analyses of the CFC and ZTPI scales with professional financial advisors. Personality and Individual Differences, 52, 723-727. 
Schneider, F. W., Gruman, J. A., \& Coutts, L. M. (2012). Applied social psychology: Understanding and addressing social and practical problems. London: Sage Publications.

Slavchov, B., Vizrmozelova, N. (2008). Osobenosti na vremevata orientaciya na studentite I izbora im na spetsialnost [Characteristics of the time orientation of the students and their choice of the university subjects] $V^{\text {th }}$ Congress of Psychology. Sofia.

Sobol-Kwapinska, M., Jankowski, T. (2016). Positive time: Balanced time perspective and positive orientation. Journal of Happiness Studies, 17 (4), 1511-1528.

Stamatov, R. (2000). Detska psihologiya [Child psychology]. Plovdiv: Hermes.

Wade, J. B., Hart, R. P., Wade, J. H., Bajaj, J. S., \& Price, D. D. (2013). The relationship between marital status and psychological resilience in chronic pain. Pain Research, and Treatment, Article ID 928473 doi:10.1155/2013/928473.

Wilson, C. M., Oswald, A. J. (2005). How does marriage affect physical and psychological health? A survey of the longitudinal evidence. Working Paper. Coventry: University of Warwick, Department of Economics. Warwick economic research papers (No.728).

Zimbardo, P., \& Boyd, J. (1999). Putting time in perspective: A valid, reliable individual differences metric. Journal of Personality and Social Psychology, 77, 1271-88.

Zimbardo, P. G., \& Boyd, J. N. (2008). The time paradox: The new psychology of time that will change your life. New York, NY: The Free Press.

Zimbardo, P. G., Boyd, J. N. (2015). Putting time in perspective: A valid, reliable individual-differences metric. In: Stolarski M., Fieulaine N., van Beek W. (Eds.), Time perspective theory; review, research, and application. Springer, Cham.

Received: October 05, 2017

Accepted: December 23, 2017
Natasha Angelova $\quad$ PhD., Chief Assistant, Professor SWU "Neofit Rilski", Blagoevgrad, Bulgaria.

E-mail: natasha_v@swu.bg 